\title{
CONDENSATION OF ZINC VAPOR IN A SUPERSONIC CARRIER GAS
}

\author{
D. D. McBRIDE, P. M. SHERMAN and T. H. PIERCE \\ Aerospace Engineering Dept. \\ The University of Michigan, \\ Ann Arbor (Michigan) U.S.A.
}

\begin{abstract}
A study of the condensation of a metal vapor in an inert carrier gas is made. Superheated zinc vapor is generated in a hot shot wind tunnel in a helium carrier gas and expanded in a converging-diverging nozzle. Static pressure measurements along the length of the nozzle are made to determine the location of the onset of condensation. A conical nominal Mach 5 (helium) nozzle is employed. The amount of supercooling before the onset of condensation is found to be approximately $430 \mathrm{~K}$, measured along the isentrope over a range of initial zinc mass fractions of .35 to .70 for saturation partial pressures of zinc between 10 psia and 70 psia. The measurements are compared with results of an analysis based on the classical liquid drop model of nucleation. The computed results agree reasonably well with the measurements.
\end{abstract}

\section{§ 1. Introduction}

Condensation is important in the analysis of many dynamic systems. Early interest was related to the condensation of water vapor in steam turbines [1], where blade erosion as well as turbine efficiency was affected by condensation. The problems become even more acute today when metal vapors are considered for the working fluid in the turbine [2]. Prevention of condensation has become an important design requirement in the extension of operation of wind tunnels to high supersonic or hypersonic velocities [3]. Stagnation temperature, for example, is usually limited to that just high enough to avoid condensation in the test section. New propulsion schemes also require a knowledge of condensation. Condensation of metal vapor is employed to produce the charge carrying 
particles of an EHD Energy Converter [4] and has been proposed as a method for producing the extremely small and numerous particles which make up the working fluid in a colloidal-electrostatic engine $[5,6]$. New designs of nuclear reactors also require a knowledge of condensation parameters. Condensation affects the limits of operation of the Kantrowitz-Grey type of molecular beam [7] and of the free jets employed for the high Mach number rarefied gas studies of shock thickness, rotational temperatures, wake flows, etc.

Condensation is also of interest in combustion problems. One example of this is in detonation waves. Quite a few years ago Kistiakowsky and Zinmann [8] noted a rise in the ChapmanJouguet Mach number of detonation for acetylene-oxygen mixtures as the fuel rich detonation limit was approached. This was determined to be due to the condensation of solid carbon. Recently Miles, Munday, and Ubbelohde [9] and Munday, Ubbelohde and Wood [10] encountered the formation of solid carbon or carbon polymers in the detonation of oxygen and furan $\left(\mathrm{C}_{4} \mathrm{H}_{4} \mathrm{O}\right)$ and oxygen and cyanogen $\left(\mathrm{C}_{2} \mathrm{~N}_{2}\right)$, respectively. There is a large energy change in this condensation process and the question remains as to whether the nucleation and condensation processes can occur rapidly enough to help support the detonation and thus alter the resultant velocities, pressures, temperatures, and species. It is reasonable to believe that similar phenomenon can occur in condensed explosives.

Condensation occurs in the transient formation of carbon particles in a hydro-carbon flame. Heterogeneous combustion will usually occur via the vapor phase. The rate of heterogeneous combustion will depend to a large degree on the rate of condensation in the system, since the latent heat liberated during condensation can be appreciable. If the rate of condensation could be predicted, the results could be used to predict: combustion efficiency, laminar burning velocity, choking of flow due to heat released, and spectral radiance of the continuum radiation emitted from hot product particles.

When a superheated vapor is expanded in a nozzle or free jet, the initial decrease in the pressure and temperature can be regarded as isentropic. In general, the vapor pressure curve (in the $\mathrm{p}-\mathrm{T}$ plane) is steeper than the isentropic curve and the expanding vapor at 
some point will become saturated (Wegener and Mack [3] give an excellent discussion of this). If the expansion is rapid and the gaseous mixture is relatively "clean", sufficient surface will not exist for the condensation necessary to maintain equilibrium $\left(p_{v}=p_{\infty}\right)$ and a supersaturated vapor $\left(p_{v}<p_{\infty}\right)$ will result. As the supersaturation continues to increase, small droplets will be formed from the vapor through a process of spontaneous (homogeneous) self nucleation [11]. As the nucleation rate increases, a point is usually reached where enough droplets exist to provide the surface necessary for the faster condensation through particle growth required to return the vapor to equilibrium, and the supersaturated state "collapses". Subsequent to this collapse a negligible number of new droplets will be formed and the existing particles will continue to grow until the supply of vapor is exhausted.

Condensation, in general, has been the subject of considerable research through the past several years. Several comprehensive reviews of both theoretical and experimental tesearch exist $[3,12$, $13,14]$. Much of the early work involved the condensation of steam $[1,15,16,17]$. However, with the observation of condensation in a supersonic wind tunnel, much of the emphasis changed to air $[18,19]$, water vapor in air [20], and nitrogen [21]. Measurements were also made in carbon dioxide [22] and ammonia vapor [23]. It is still not possible however, to predict condensation conditions for any given system. There is a dearth of both experimental and theoretical information.

The purpose of this paper is to present new measurements and computations made for the condensation of metal vapor in an inert carrier gas [24].

\section{§ 2. Experimental arrangement and procedure}

One problem which arises in the study of metal vapor is the production of the extreme conditions of temperature (at reasonable pressure), necessary to obtain the superheated conditions required. The choice of metal vapor and carrier gas therefore depended largely upon their suitability for laboratory measurements. Toxity and high chemical activity made the use of some of the metals unfeasible. Zinc was chosen as one metal with fewer difficulties than most. It has a saturation temperature at reasonable pressures that is not inordinately high and it has physical properties which 
are well behaved in both gaseous and condensed phases. It has constant, or near constant, specific heats, ratio of specific heats, and latent heat along with well known and reliable physical data (such as vapor pressure) which make analysis of results much easier and comparison of theory and experiment much more meaningful (since fewer approximations must be made). The low vaporization temperature meant that it would remain superheated at lower temperatures and thus operation problems would be minimized by minimizing energy transfer into the stagnation chamber. Zinc has the added advantage of structural strength and convenient handling and machining properties which made possible the use of the test metal as part of the arc chamber electrode arrangement as explained below.

The metal vapor was generated in the University of Michigan hot shot wind tunnel facility [25]. The tunnel is a blowdown type of facility. It makes use of energy initially supplied by a $150 \mathrm{HP}$ motor and stored over a "long" period of time as kinetic energy in a heavy flywheel. The stored energy is transferred to a magnetic field of a large coil by means of a unipolar generator designed to deliver high current at low voltage. The energy is discharged through an arc across electrodes inside the high pressure stagnation chamber. The arc is initiated by means of a zinc fuse switch arrangement which was redesigned [24] so that the desired amount of zinc vapor would be obtained during the arcing process. The stagnation chamber was initially charged with the desired amount of helium so that when arcing accured, the transfer of energy at constant volume raised the temperature and pressure of the vapor to the desired superheated state. The resultant mixture of superheated zinc vapor and helium was expanded through a nominal Mach 5 nozzle to room conditions. The tunnel is capable of storing up to approximately $1.5 \times 10^{7} \mathrm{in}$.- $\mathrm{lb}$ of energy in the energy storage coil. The energy is transferred to the working fluid at efficiencies of the order of $50 \%$.

Helium was chosen as a carrier gas because it is light, inert, monatomic and will not condense at the temperatures commonly reached in the laboratory.

The specific energy, $e$, necessary to heat, at constant volume, a mixture of metal and gas from a charge temperature $T_{c h}$, to a final temperature, $T_{f}$, vaporizing the metal in the process, can be 
approximated by:

$$
e=C_{v_{c}}\left(T_{f}-T_{c h}\right)+(1-f) L_{c h}
$$

where $L_{c h}$ is the latent heat of vapor defined at charge condition and the mixture constant volume specific heat $C_{v_{c}}$ is defined by:

$$
C_{v_{a}}=f C_{v_{a}}+(1-f) C_{v_{v}}
$$

where $f$ is the mass fraction of carrier gas, and $C_{v_{a}}$ and $C_{v_{v}}$ are, respectively the carrier gas and vapor specific heats.

For a given volume $V$, the total energy $E$ required to reach a final pressure $p_{f}$ and temperature $T_{f}$ is

$$
E=\frac{p_{f} V e}{R T_{f}} \frac{\mu_{v}}{1-f+f \alpha}
$$

where $V$ is total volume, $\mu_{v}$ is the molecular weight of the vapor, $\alpha$ is the ratio of the molecular weight of the vapor to the molecular weight of the carrier gas and $R$ is the universal gas constant.

The total energy required to raise a zinc-helium mixture from $298 \mathrm{~K}$ to $5000 \mathrm{~K}$, and 5000 psia in a volume of 65 in. ${ }^{3}$ (tunnel arc chamber) is shown in Fig. 1 as a function of helium mass fraction $f$.

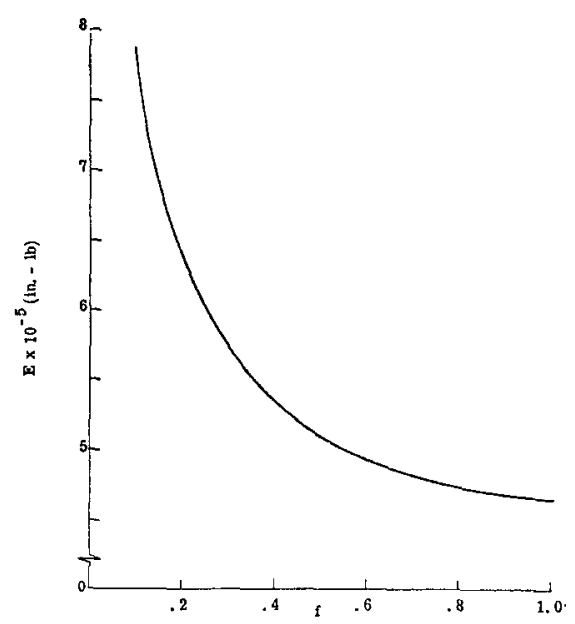

Fig. 1. Energy required for production of a zinc-helium mixture at $5000 \mathrm{~K}$ and 5000 psia as a function of mass fraction of helium with an initial temperature of $298 \mathrm{~K}$. 
It can be seen that the amount of energy required for moderate operating conditions, lies between $4 \times 10^{5}$ and $8 \times 10^{5}$ in.-1b depending upon the amount of zinc vaporized. Thus, the hot shot is easily capable of furnishing the energy for the operating conditions necessary to obtain the required superheated zinc vaporhelium mixture. In addition, the hot shot provided a ready means of introducing the zinc vapor into the flow by means of the arcing process employed to transfer the energy from the main coil to the working fluid.

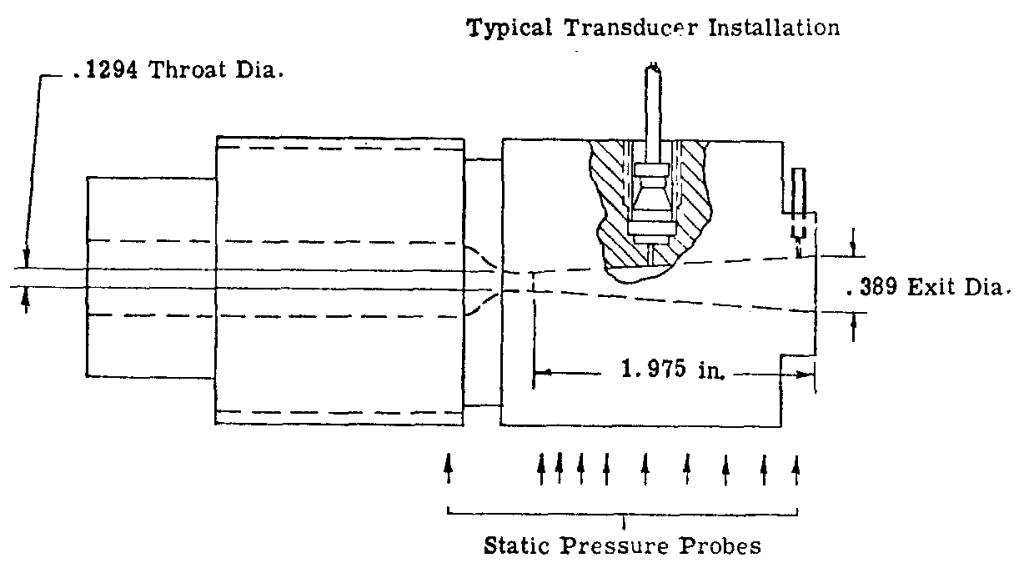

Fig. 2. Schematic of mach 5 nozzle.

A Mach 5 (helium) nozzle (Fig. 2) was designed for making static pressure measurements and was fabricated from beryllium copper. This nozzle has a .1294 in. diameter throat, a total included angle of $7.45^{\circ}$, and a .389 in. exit diameter. These dimensions were chosen, (1) to give a reasonable blowdown rate, (2) to provide sufficient space for the transducer mountings while keeping the nozzle as short as possible to minimize boundary layer, and (3) to maintain underexpansion when exhausting to atmosphere in order to minimize the chance of boundary layer separation near the nozzle exit. Nine static pressure taps are located along the 2 in. length of the supersonic portion according to Table I. A stagnation pressure tap is located jusr upstream of the throat at a diameter of $.500 \mathrm{in}$. Stagnation pressure was also measured at the arc chamber. 
TABLE I

\begin{tabular}{cc}
$\begin{array}{c}\text { Location of Pressure Ports in Nozzle } \\
\text { Distance from Throat } \\
\text { (inches) }\end{array}$ & $\begin{array}{c}\text { Geometric Area } \\
\text { Ratio }\end{array}$ \\
\hline 0.0547 & 1.1132 \\
0.1897 & 1.4188 \\
0.3522 & 1.8358 \\
0.5336 & 2.3645 \\
0.7968 & 3.2505 \\
1.1038 & 4.4616 \\
1.3712 & 5.6722 \\
1.8589 & 8.2545 \\
\hline
\end{tabular}

Most measurements and timing signals were recorded on a 50 channel CEC recording oscillograph Model S-119, at a recording speed of 100 in. per sec. Stagnation chamber pressure was measured with a Baldwin-Lima-Hamilton GP 10,000 psi bonded strain gage pressure transducer or a Kistler Model 603H 15,000 psi piezoelectric transducer. The stagnation pressure measurement made just upstream of the nozzle throat was with a Schaevitz-Bytrex Model HFH 500 psi semi conductor strain gage transducer. Pitot pressure at the nozzle exit was measured with a Kistler 601 piezoelectric transducer. Pressures at the seven locations at the upstream end of the nozzle were measured with Microsystems Model 1003 pressure transducers. These transducers are of the flush diaphragm type with semiconductor strain gages bonded directly to the diaphragm in a fullbridge circuit. The rise time of these transducers is in the $\mu \mathrm{sec}$ range. They were all driven by carrier amplifiers. The pressures at the two downstream locations were measured with Hidyne $W$ Series 30 psid transducers of the variable reluctance type. These transducers are very rugged and versatile with a rise time of less than $1 \mathrm{msec}$ at the pressure encountered in this experiment. They also were driven by carrier amplifiers. A constant reference pressure of approximately 15 psig was applied to these transducers to increase their null or zero level to cause the transducers to operate closer to 0 psid in a more linear range.

All of the strain gage transducers were calibrated on a dead weight tester (Ashcroft Model 1305-B-50) and reference calibration signals were applied to the amplifiers so that they could be applied to the 
calibration of the amplifier system by itself. The variable reluctance gages were calibrated by applying pressure to the "high" side of the transducer and measuring the pressure with a calibrated bourdon tube gage. The Hidyne transducers had the higher system calibration error - error of $\pm 5 \%$ of full scale reading. All the other transducer calibrations were considerably more repeatable.

Since it has been shown $[3,24]$ that approach to equilibrium is fast after supersaturation collapses, flow with equilibrium condensation was compared analytically with flow downstream of onset of condensation in nonequilibrium flow to confirm that some distance downstream of onset the equilibrium solution is a good approximation to the actual flow. This assumption was then used to check the mass fraction and stagnation temperature in the arc chamber. If it is assumed that equilibrium has been reached at two of the more downstream pressure taps, then both of these pressures will fit only one equilibrium expansion curve. This expansion 7ives a unique stagnation temperature and zinc concentration. The procedure used to determine this curve is outlined below.

For one of the measured static pressures several different reasonable stagnation temperatures were selected. For each temperature, the equilibrium expansion was calculated [26] for varying zinc concentration until the concentration was found which caused the expansion curve to fit the measured pressure. This gave a locus of stagnation temperature-zinc concentration combinations whose equilibrium expansion curves all passed through the given pressure ratio. The same procedure was followed for the other measured pressure and the intersection of the two curves yielded the unique stagnation temperature and zinc mass fraction.

The onset of condensation was determined by the location in the nozzle at which the static pressure measured deviates from the value for the "frozen" (without condensation) flow expansion. It was previously shown $[24,27]$ that the nozzle effective area ratio may be determined by pitot tube measurements for the low supersonic Mach numbers used here. Static pressures were therefore nondimensionalized by chamber pressure and plotted against area ratio determined from pitot tube measurements. A comparison of these measured pressure ratios was then made with the pressure ratios for flow with no condensation. Fig. 3 is an example of such a plot. 


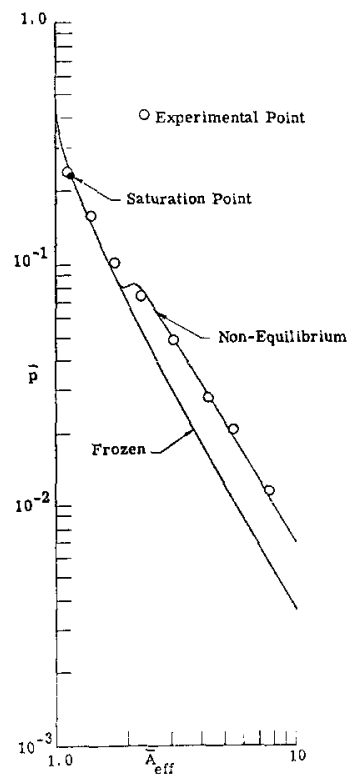

Fig. 3. Ratio of static pressure to stagnation chamber pressure, $\bar{p}$ as a function of effective area ratio. (Stagnation chamber temperature, pressure, mass fraction of carrier gas are $2273 \mathrm{~K}, 1772$ psia, 0.4, respectively.)

\section{$\S 3$. Results, analysis and discussion}

As mentioned previously, the measurement of static pressure was used to determine the location of condensation onset in the nozzle. Fig. 3 shows a typical expansion curve obtained during the study. The static pressure (nondimensionalized by the chamber pressure) is plotted against effective area ratio. The "frozen" isentropic expansion and an analytical nonequilibrium solution [24] are shown for comparison. As can be seen, the location of the onset point cannot be determined more accurately than the pressure tap spacing. In this case, it can only be said that onset occurs between an area ratio of 1.76 (where the experimental point still lies on the "frozen" expansion curve) and 2.26 (where the experimental point has definitely departed in the direction of the equilibrium curve). These two outside limits correspond to pressure ratios of 0.090 and 0.054 or, using Dalton's Law (at onset no condensation has yet taken place), to zinc partial pressures $p_{z}$ of 12.9 and 7.7 psia.

While a pressure versus area ratio plot is fine for studying the 
manner in which onset occurs, pressure-temperature coordinates are of interest in the comparison of results of different expansions. The nozzle expansion of the zinc vapo: before condensation may be considered isentropic and as such, $p_{z}$ vs $T$ is represented by a straight line of slope $(\gamma / \gamma-1)$ when plotted on a log-log scale. The position of the line is determined by the chamber conditions, $p_{c_{z}}$ and $T_{c}$. Fig. 4 shows a summary of the onset data obtained for zinc vapor in helium.

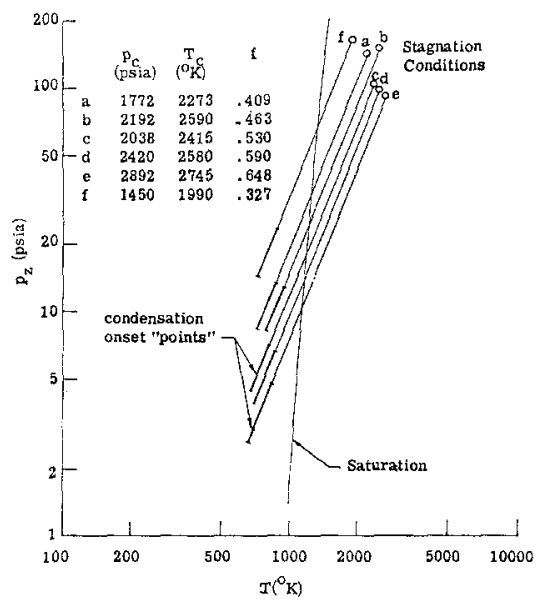

Fig. 4. Partial pressure of zinc, $p_{z}$, as a function of local temperature for Several sets of stagnation conditions showing region of onset of condensation.

Within the accuracy the apparatus dictates, the onset "points" seem to yield a definite onset curve which is characterized by an almost constant separation (at constant pressure) from the saturation curve. A common method of defining the amount of supersaturation at onset is by means of the "supersaturation temperature decrement" $\Delta T$, defined as the difference between the temperature at which the flow isentrope crosses the saturation curve and the temperature at which onset of condensation occurs. One interesting feature of the measurement is that $\Delta T$ for the conditions considered here is found to be almost constant at $430 \mathrm{~K}$ over the range of conditions considered. The onset points computed are also shown and indicate reasonable agreement. Similar results were obtained 
using argon [28] where the $\Delta T$ found appeared to bc approximately $20 \mathrm{~K}$ over a range of argon saturation point pressures between 7 psia and 300 psia.

An analysis was made using a previously developed computer program [30]. The program was based on the classical liquid drop theory for nonequilibrium condensation with the usual assumptions [30]. While having been under increasing attack recently, the classical theory for nonequilibrium condensation still seems to have the greatest "success" in matching experimental data from nozzle flows $[3,29,31]$. It provides a basis for comparison with, and extrapolation of, experimental data. It also provides in a qualitative manner prediction of the effects of different flow parameters on the condensation process. The liquid drop nucleation model yields a nucleation rate equation in terms of a "critical" drop size. The value of the surface tension was taken as a function of drop size [32] through a constant which was originally related to the intermolecular distance in the liquid drop. Although there is some disagreement on surface tension correction for small radii, this correction seems to be generally regarded as the best available $[2,34]$ and is somewhat substantiated by experiment [35]. Particle size measurements previously made [24] were used to approximate the Tolman constant and proved to be within the appropriate limits.

The growth rate equation used for comparison with the present experimental results was one used by Hill [31] with the following two approximations: (1) the droplet temperature was assumed equal to the equilibrium saturation temperature $T_{\infty}$ corresponding to the vapor partial pressure, and (2) the droplet pressure was assumed equal to the vapor partial pressure (i.e., the droplet is considered to be large enough to allow neglect of the surface tension contribution).

Fig. 5 shows the same set of onset data as in Fig. 4 with constant nucleation rate curves and computed onset points superposed. Quite some time ago, Head [20] proposed that the attainment of an approximate nucleation rate of $J \doteq 10^{18}$ (drops/in. ${ }^{3}$-sec) typifies the onset of condensation in water vapor. He justified this argument on both theoretical and experimental grounds. More recently, it has been shown [29] that this figure also serves to indicate onset in nitrogen and that calculations indicate the figure may be good for pure copper vapor also. The onset point in zinc vapor expanding 


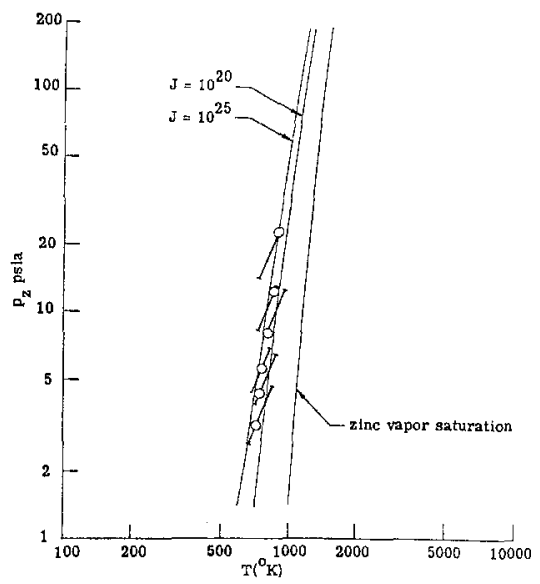

Fig. 5. Partial pressure of zinc as a function of local temperature for constant nucleation curves, saturation curve, computed of location condensation onset (circles) and measured onset regions.

in helium carrier gas however, seems to be typified by a nucleation rate between $10^{20}$ and $10^{25}$ (drops/in. ${ }^{3}$-sec.).

It has long been known that ionized particles will act as condensation nuclei in a supersaturated vapor. This is the underlying principle of the Wilson Cloud Chamber. Thus, due to the relatively low ionization potentials of metals and the very high temperatures reached in the arc chamber, the possible effect on condensation of ionization in the arc chamber is certainly a question which cannot be overlooked. Solbes and Kerrebrock [33] have shown that non-equilibrium ionization effects on nucleation and condensation are minor in potassium at temperatures below $2000 \mathrm{~K}$ at 1 atmosphere. A simple equilibrium ionization calculation on potassium vapor at these conditions yields an ionized mass fraction of $2.7 \times 10^{-5}$. Due to the much higher ionization potential of zinc, stagnation conditions of 4650 psia and $4650 \mathrm{~K}$, at a zinc mass fraction of 0.75 , are necessary to obtain a comparable ionization level. However, all of the measurements of condensation onset were made at stagnation pressures and temperatures approximately half that value. At the typical chamber conditions of 2500 psia, $2500 \mathrm{~K}$, and 0.75 zinc mass fraction, the ionized mass fraction of zinc is only $6.8 \times 10^{-10}$. Thus the equilibrium ionization level at 
stagnation conditions is five orders of magnitude less than an equilibrium level would be in the potassium case. Since the higher density in the zinc flow would tend to minimize the non-equilibrium ionization downstream of the chamber, it seems reasonable that ionization, in toto, can be assumed to be negligible. Earlier in the run when the temperatures are high and the arc is still established, it is not clear what the ionization level would be.

It is felt that contamination did not play a strong role in influencing any of the major findings of this study. The fact that the heavily eroded center electrode was made of zinc and that there was no evidence that the other end of the arc column established itself at any one point (other than the zinc fuse) would lend credence to the assumption that the only significant "contaminant" was zinc (it should be noted that hotshot contamination usually derives from only one electrode and the fuse, which were made of pure $(99.99+\%)$ zinc in this case). Further, considerable supersaturation of zinc vapor during the expansion indicates negligible effects from contamination since contamination would have tended to cause a saturated expansion.

\section{Acknowledgment}

This research was supported in part by the Aerospace Research Labs, Office of Aerospace Research USAF under contract AF $33(615)-67 c-1197$.

Received 26 October 1970

\section{REFERENCES}

[1] Stodola, A., Steam and Gas Turbines, McGraw-Hill Book Company, New York, 1927.

[2] Hili, P. G., H. Witting and E. P. Demetri, J. of Heat Transfer, 85 (1963) 303-317.

[3] Wegener, P. P. and L. M. Mack, Adv. in Appl. Mech., Vol. V, Academic Press, 1958, pp. 307-447.

[4] Cox, A. L., AIAA J., 1 (1963) 2491-2497.

[5] Goldin, D. S., A Thermodynamic Flow Analysis of Particle Formation Efficiency in a Mixed Flow Colloid Thrustor, AIAA Paper No. 67-85, 5th Aerospace Sciences Meeting, New York, January 1967.

[6] Norgren, C. T., Progress in Astronautics and Aeronautics, 9, Academic Press, New York, (1963) 407-434.

[7] BosseL, U., Investigation of Skimmer Interaction Influences on the Production of Aerodynamically Intensified Molecular Beams, Report No. AS-68-6, University of California, Berkeley, 1968. 
[8] Kistiakowsky, G. B. and W. B. Zinmann, J. Chem. Phys., 23 (1955) 1889.

[9] Mrles, J. E. P., G. Munday and A. R. Ubbelohde, Proc. Roy. Soc. A, 269 (1962) 165.

[10] Munday, G., A. R. Ubbelohde and I. F. Wood, Proc. Roy. Soc. A, 306 (1968) 179.

[11] Frenkel, J., Kinetic Theory of Liquids, Clarendon Press, Oxford, 1956, pp. 366400. (Also published by Dover Publications, Inc., New York.)

[12] Stever, H. G., High Speed Aerodynamics and Jet Propulsion, Vol. III, Princeton University Press, Princeton, N.J., 1958, pp. 526-573.

[13] Courtne, Y, W. G., Condensation During Heterogeneous Combustion, Eleventh Symposium (International) on Combustion, The Combustion Institute, Pittsburgh, Pa., 1967, pp. 237-244.

[14] Courtney, W. G., Re-examination of Nucleation and Condensation of Water, Techn. Rept. Thiokol Chemical Corporation, Reaction Motors Division, Denville, N.J., July 1964.

[15] Binnie, A. M. and J. R. Green, Proc. Roy. Soc. A, 181 (1943) 134.

[16] Binnie, A. M. and M. W. Woods, The Pressure Distribution in a ConvergentDivergent Steam Nozzle, Proc. Inst. Mech. Engrs., No. 138, London, 1938, p. 229.

[17] Yellott, J. I., Engineering, 137 (1934) 303.

[18] BUHzER, R. D., Condensation of Air Components in Hypersonic Wind Tunnels. Theoretical Calculations and Comparison with Experiment., Ph.D. Thesis, California Institute of Technology, Pasadena, California, 1952.

[19] DaUm, F. L., AIAA J. 1 (1963) 1043-1046.

[20] HEAD, R. M., Investigations of Spontaneous Condensation Phenomena, Ph.D. Thesis, California Institute of Technology, Pasadena, California, 1949.

[21] Willmarth, W. W. and H. T. Nagamatsu, Condensation of Nitrogen in a Hypersonic Nozzle, GALCIT Hypersonic Wind Tunnel Memorandum No. 6, January, 1952.

[22] Duff, K. M., Non-Equilibrium Condensation of Carbon Dioxide in Supersonic Nozzles, Ph.D. Thesis, Massachusetts Institute of Technology, Cambridge, Mass., January 1966.

[23] Kremmer, M. and O. OKurounmu, Condensation of Ammonia Vapor During Rapid Expansion, Gas Turbine Laboratory Report No. 79, Massachusetts Institute of Technology, 1965.

[24] Sherman, P. M., D. D. McBride, T. Chmielewsiki, T. H. Pierce and E. Oktay, Condensation of Metal Vapor in a Supersonic Carrier Gas, OAR Report No. ARL 69-0089, U.S.A.F. June 1969.

[25] Sherman, P. M., Development and Operation of an Arc Heated Hypersonic Tunnel, Report 02953-3-F, University of Michigan, July 1963.

[26] McBride, D. D. and P. M. Sherman, Astronautica Acta 16 (1971) 97.

[27] McBride, D. D. and P. M. Sherman, Measurement of Pitot Pressure in Flow with Condensation, accepted for publication in AIAA J., 17 (1971) 97.

[28] Pierce, T. H., P. M. Sherman and D. D. McBride, Astronautica Acta.

[29] Griffin, J. L. and P. M. Sherman, AIAA J., 3 (1965) 1813.

[30] Chmielewsi, T. and P. M. Sherman, AIAA J., 8 (1970) 789.

[31] Hilr, P. G., J. of Fluid Mechanics, 25 (1966) 593-620.

[32] Tolman, R. C., J. Chem. Phys., 17 (1949) 333-337.

[33] Solbes, A. and J. L. Kerrebrock, Physics of Fluids, 10 (1967) 2179.

[34] ThomanN, H., Determination of the Size of Ice Crystals Formed During Condensation of Water in Wind Tunnels and of Their Effect on Boundary Layers, FFA Report No. 101, The Aeronautical Research Institute of Sweden, Stockholm, July 1964.

[35] Stein, G. D. and P. P. Wegener, J. Chem. Phys., 46 (1967) 3685. 